\title{
Decision to enroll in post-graduate education: What are the predictors?
}

\begin{abstract}
The objective of the study was to predict students' decision to enroll for a doctoral program at the Faculty of Educational Studies. A discriminant analysis was conducted to investigate the predictors that influence a student's decision to enroll for a doctoral program. The predictors investigated were job factors, personal factors, program factors, social factors, satisfaction, and academic performance. The overall Wilk's Lambda was significant, $\Lambda=0.87$, ChiSquare $(6, \mathrm{n}=117)=15.99, \mathrm{p}=0.014$. This indicated that the predictors were significantly different among groups of student who plan to enroll for a doctoral program (Group 1) and those who do not (Group 2). Of the total 117 students, 90 (76\%) were classified correctly.
\end{abstract}

Keyword: Post-graduate dducation, predictors, continuing education 